\title{
The role of physical and chemical processes of silica scale growth in geothermal wells
}

\author{
Akihiro MIZUSHIMA ${ }^{1}$, Hitoshi MIKADA ${ }^{2}$ and Junichi TAKEKAWA ${ }^{2}$ \\ ${ }^{1}$ Dept. of Civil and Earth Res. Eng., Kyoto University (Now at Ministry of Land, Infrastructure and Transport) \\ ${ }^{2}$ Dept. of Civil and Earth Res. Eng., Kyoto University
}

\begin{abstract}
Silica scaling remains to be a major restriction for geothermal heat extraction. Our goal is to construct the model reproducing the real silica scaling. To meet this goal, we develop the multi-scale modeling of silica scale growth and compare the simulation result of the amount and the distribution of silica deposition and the data from a laboratory or a field experiment to verify our model. In meso-scale model, the adhesion of the colloidal silica is analyzed using Lagrangian method, while, in the macro-scale model, LB simulation is performed using the scale growth rate obtained at the meso-scale model. From our simulation result, the real phenomenon is reproduced quantitatively and quantitatively, which has not been reproduced in the reaction kinetics. It is, therefore, necessary to emphasize the adhesion of the colloidal silica should be taken into account for reproducing silica scaling.
\end{abstract}

\section{INTRODUCTION}

Silica scaling in pipe systems, including heat exchangers, is one of the most significant problems in the geothermal power plants. The deposited material accumulates on the inside of pipes and reduces the effectiveness of utilization of the geothermal resources by clogging pipes. A similar problem is faced in the oil \& gas industry with calcium carbonate scaling ${ }^{1)}$.

Geothermal fluid at high temperature and pressure is in chemical equilibrium with the surrounding rock. The temperature and pressure of this fluid decreases as it is extracted from the reservoir and cycled through the pipe systems. Silica concentration then exceeds the amorphous silica solubility leading to precipitation. These colloidal silica particles are transported to pipe surfaces and gradually accumulate.

There have been a number of experimental studies on the kinetics of silica scale formation ${ }^{2-5}$. Although they determined the kinetic parameter using both experimental data and derived the kinetics of silica dissolution- precipitation from theoretical considerations, it has been reported that the magnitude of deposition rate is extremely lower than that in the fields ${ }^{6,7)}$.

A high rate growth of scale is often observed where the flow stagnates ${ }^{8}$. The inhomogeneous flow could influence the scale growth, which is an alternative process by which the colloidal silica is transported to a surface and adhere the surface. However the mechanism has not been well understood.

We construct the multi-scale model of the silica scaling considering the particle deposition of colloidal silica on the pipe wall in order to evaluate the hydrodynamical effect quantitatively. Our findings are compared with two observation in the literature ${ }^{9,10)}$ to verify our model.

\section{NUMERICAL METHODS}

The multi-scale modeling of silica scale growth is developed. The scale growth rate in the macro-scale model is determined from the analysis based on the meso-scale model.

\section{(1) Particle motion}

In the meso-scale model, the trajectory of a particle near the wall surface is calculated by solution of the linear momentum equations ${ }^{11-13)}$

$$
m \boldsymbol{u}_{p}=\boldsymbol{F}_{D}+\boldsymbol{F}_{B G}+\boldsymbol{F}_{E L E}+\boldsymbol{F}_{V d W},
$$

where $m$ is the particle mass, $\boldsymbol{u}_{p}$ is the particle velocity and $\boldsymbol{F}_{D}$ is the drag force, $\boldsymbol{F}_{B G}$ the buoyancy force and gravity, $\boldsymbol{F}_{E L E}$ the electrostatic force and $\boldsymbol{F}_{V d W}$ the van der Waals force.

\section{(2) Lattice Boltzmann method (LBM)}

We use the lattice Boltzmann method to simulate fluid flow. In the lattice Boltzmann model, the two-demensional nine-directional (D2Q9) ${ }^{14)}$ lattice 
Bhatnager-Gross-Krook model (LBGK) ${ }^{15)}$ is set. In this model, the directions of the discrete velocity are given as

$$
c_{i}=\left\{\begin{array}{cc}
c\{\cos (i \pi / 4), \sin (i \pi / 4)\} & \text { for } i=0,2,4,6 \\
\sqrt{2} c\{\cos (i \pi / 4), \sin (i \pi / 4)\} & \text { for } i=1,3,5,7 \\
(0,0) & \text { for } i=8
\end{array}\right.
$$

where $c=\Delta \boldsymbol{x} / \Delta t$ is the particle speed. The evolution equation of the particle velocity distribution function $f_{i}(\boldsymbol{x}, t)$ reads

$$
f_{i}(\boldsymbol{x}+\Delta \boldsymbol{x}, t+\Delta t)-f_{i}(\boldsymbol{x}, t)=-\frac{1}{\tau}\left[f_{i}(\boldsymbol{x}, t)-f_{i}^{e q}(\boldsymbol{x}, t)\right],
$$

where $\Delta \boldsymbol{x}$ and $\Delta t$ are the lattice spacing and the time step respectively. The relaxation time $\tau$ is a parameter which characterizes the constitutive behavior of the fluid considered at a macroscopic level. It is related to the macroscopic kinetic viscosity $v$ of the simulated fluid as

$$
v=\frac{c^{2} \Delta x}{3}\left(\tau-\frac{1}{2}\right) \text {. }
$$

$f_{i}^{e q}(\boldsymbol{x}, t)$ is the local equilibrium distribution function. In order to recover the correct Navier-Stokes equations, the local equilibrium distribution function is defined as

$$
f_{i}^{e q}=\omega_{i} \rho\left[1+3 \frac{\left(\boldsymbol{c}_{i} \cdot \boldsymbol{u}\right)}{c^{2}}+\frac{9}{2} \frac{\left(\boldsymbol{c}_{i} \cdot \boldsymbol{u}\right)^{2}}{c^{4}}-\frac{3}{2} \frac{\boldsymbol{u}^{2}}{c^{2}}\right],
$$

where $\omega_{i}$ represents the weight factors, $\rho$ is the flow density and $\boldsymbol{u}$ is the flow velocity vector given as follow:

$$
\rho=\sum_{i=0}^{8} f_{i}(\boldsymbol{x}, t), \boldsymbol{u}=\sum_{i=0}^{8} f_{i}(\boldsymbol{x}, t) \cdot \boldsymbol{c}_{i} \cdot
$$

We describe the transport of colloidal silica macroscopically with the convection-diffusion equation (CDE) written as

$$
\frac{\partial C}{\partial t}+(\boldsymbol{u} \cdot \nabla) C=D \nabla^{2} C
$$

where $C$ is the concentration and $D$ is the diffusion ciefficient. In this paper, the mass transfer for LB model adopts D2Q5 model ${ }^{16)}$, which is different from D2Q9 models but any real loss of accuracy. The D2Q5 model can be successfull used to simulate the cause with very small $D$ by adjusting $\lambda$ without any loss of simulation stability ${ }^{17}$.

We apply the algorithm of the deposition used in the LB simulation of crystal growth ${ }^{18,19)}$ or soot deposition $^{20,21)}$. Each node at the interface represents a control volume with a size of $\Delta x \times \Delta x$ (in lattice units) and is located at the center of this control volume. We update the volume ratio of silica scale based on the growth rate. When it exceeds 1, a nearest neighboring fluid solid becomes a solid node.

\section{RESULTS}

\section{(1) Derivation of scale growth rate}

The growth rate at macro-scale is derived from meso-scale model of particle adhesion on the wall surface. We consider the case of a spherical colloidal silica particle moving parallel to a vertical or horizontal wall in a wall-bounded linear shear flow. Our simulation is performed on any $H$ to obtain constitutive expressions of threshold separation from the particle center to the wall $H$ and the time up to adhesion $T$, using the shear rate $S$ and the length of the interest area $X$. (Fig. 1) The growth rate of silica scale $R^{\mathrm{CC}}$ at the control cell $(\Delta x \times \Delta x)$ is given as

$$
R^{\mathrm{CC}}=\frac{\rho(H \Delta x) C}{T}
$$

\section{(2) Quantitative verification of macro-scale model}

Hosoi and Imai (1982) carried out an experiment of the artificial silica formation in the laboratory. We predict the amount of silica deposition on the glass wall surface to compare directly with the experiment ${ }^{9)}$ using the deposition rate determined by the analysis based on the meso-scale model. As a result, the predicted amount of surface deposition is consistent in amount as compared with that observed by the experiment ${ }^{9)}$ (Table. 1). The mass of surface deposition estimated by the general reaction kinetics has the magnitude extremely lower than the amount of laboratory experiment ${ }^{9)}$.

\section{(3) Scale profile in geothermal well}

In the production well, a higher rate of silica scale growth is observed where the flow stagnates (Fig. $)^{10)}$. To reproduce that feature and investigate the accuracy of our model, the prediction of silica scale growth in model B (Fig. 3) is performed. Our scale profile had good agreement with an observation in the geothermal well ${ }^{10)}$ as shown Fig. 4.

\section{Conclusion}

We develop the multi-scale physicochemical model considering the colloidal silica deposition for correctly predicting silica scale formation. The transient simulation of silica scale growth is performed using the lattice Boltzmann method. We quantitatively and qualitatively produce the silica scale formation in our simulations, which has not been reproduced in the reaction kinetics. We would like to emphasize that the colloidal silica deposition should be taken into account for reproducing the silica scale growth. We also conclude that shear rate is a significant factor determined the scale growth 


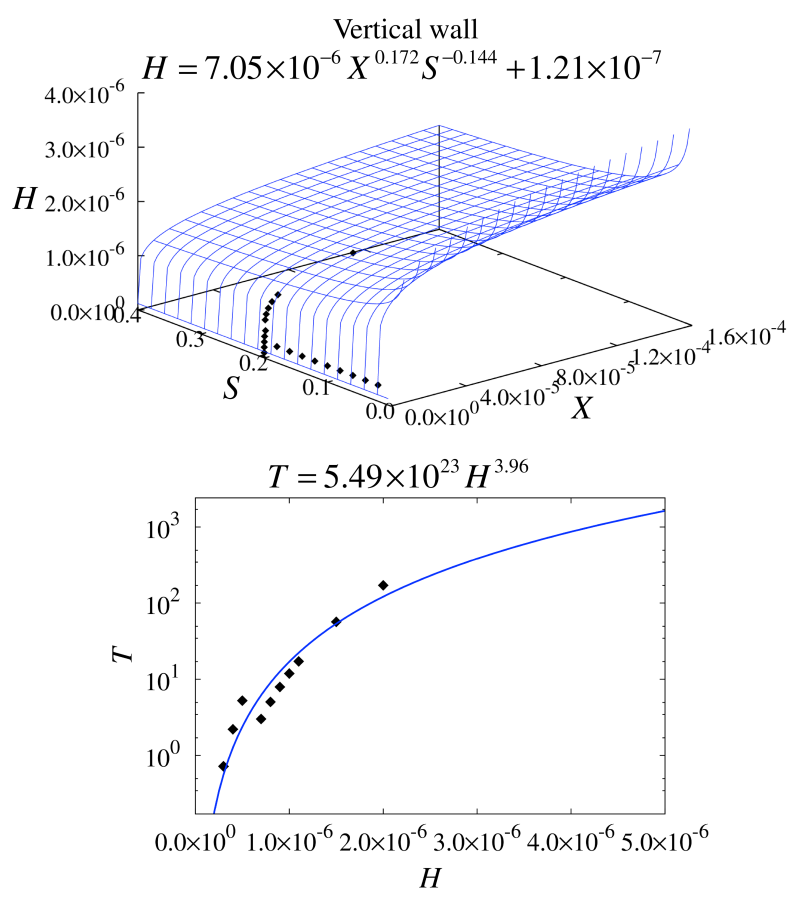

Figure 1 threshold separation as a function of shear rate and length of interest area and time up to adhesion as that of threshold separation.

Table 1 Mass of surface silica deposition $24 \mathrm{~h}$ after initiation of calculation.

\begin{tabular}{cc}
\hline & Mass of surface silica deposition $\left[\mathrm{g} / \mathrm{m}^{2}\right]$ \\
\hline Our result & 1.20 \\
\hline \hline Hosoi and Imai (1982) & $0.42-1.7$ \\
Chemical kinetics & $5.18 \times 10^{-5}$ \\
\hline
\end{tabular}
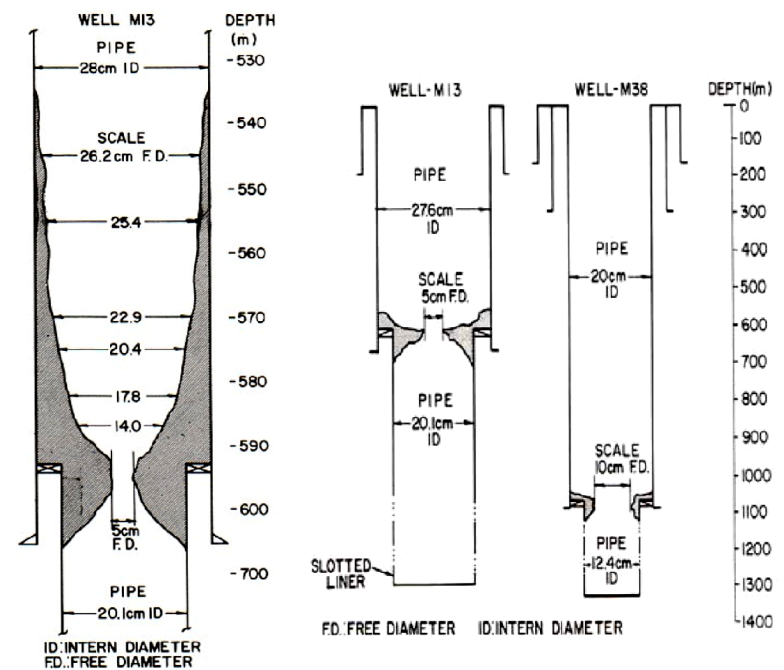

Figure 2 Scale profile in geothermal well ${ }^{10)}$

rate.

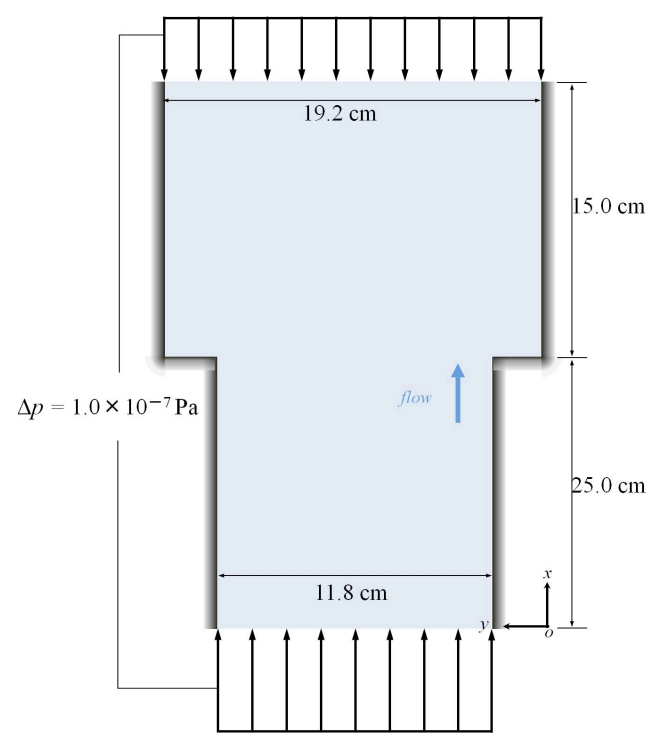

Figure 3 model B (initial condition)

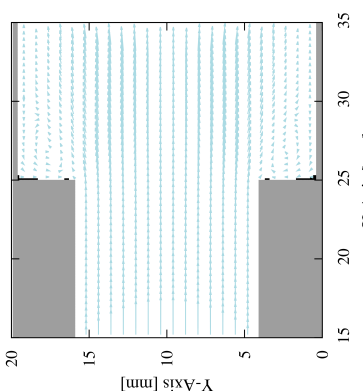

(a) $80 \mathrm{mg} / 18.8$ months

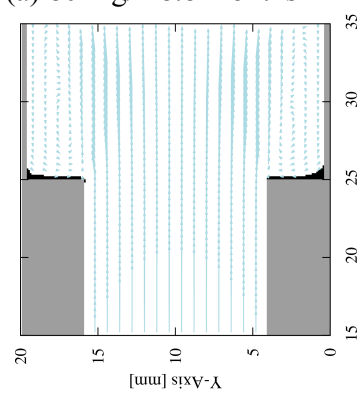

(c) $400 \mathrm{mg} / 184$ months

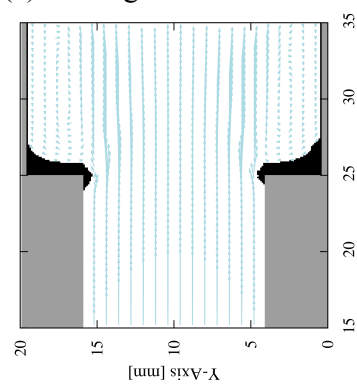

(e) $2.4 \mathrm{~g} / 747$ months

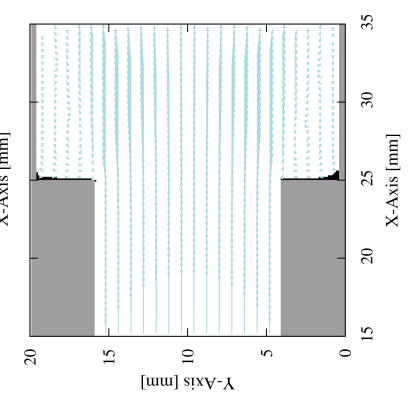

(b) $240 \mathrm{mg} / 116$ months

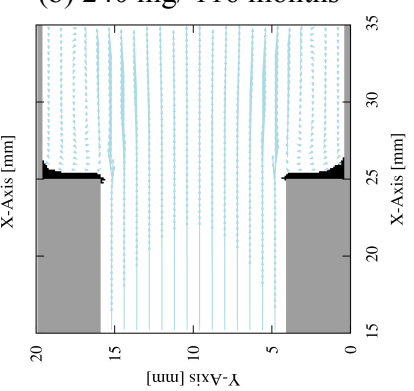

(d) $800 \mathrm{mg} / 367$ months

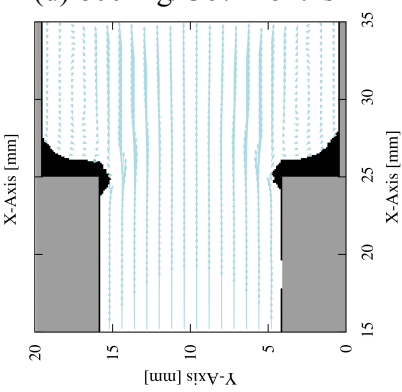

(f) $4.0 \mathrm{~g} / 943$ months
Figure 4 Time change of build-up of silica scale 


\section{REFERENCES}

1) Zhang, Y., Shaw, H., Farquhar, R. and Dawe, R., 2001, The kinetics of carbonate scaling - application for the prediction of downhole carbonate scaling, Journal of Petroleum Science and Engineering, 29(2), 85-95.

2) Rimstidt, J. D., and H. L. Barnes, 1980, The kinetics of silica-water reactions, Geochimica et Cosmochimica Acta, 44,. 1683-1699.

3) Bohlmann, E. G., R. E. Mesmer, and P. Berlinski, 1980, Kinetics of silica deposition from simulated geothermal brines, Journal of Petroleum Science and Engineering, 20, 239-248.

4) Bird, G., J. Boon, and T. Stone, 1986, Silica transport during steam injection into oil sands I. Dissolution and precipitation kinetics of quartz--new results and review of existing data, Chemical Geology, 54, 69-80.

5) Fleming, B. A., 1986, Kinetics of reaction between silicic acid and amorphous silica surfaces in $\mathrm{NaC} 1$ solutions, Journal of Colloid and Interface Science, 110, 40-64.

6) Malate, R. C. M., and M. J. O'Sullivan, 1992, Mathematical modeling of silica deposition in a porous medium, Geothermics, 21(3), 377-400.

7) Weir, G. J., and S. P. White, 1996, Surface deposition from fluid flow in a porous medium, Transport in Porous Media, 25, 79-96.

8) Garibaldi, F. (1980), The effect of some hydrodynamic parameters on silica deposition, Diploma Project 80.11, Geothermal Institute, University of Auckland.

9) Hosoi, M. and Imai, H. (1982), Study on precipitation and prevention of the silica scale form the geothermal hot water, Journal of the Geothermal Research Society of Japan, 4, 127-142.

10) Mercado, S., Bermejo, F., Hurtado, R., Terrazas, B. and Hernandez, L. (1989), Scale incidence on production pipes of Cerro Prieto geothermal wells, Geothermics, 18(1), 225-232.

11) Apostolou, K. and Hrymak, A. N. (2008), Discrete element simulation of liquid-particle flows. Computers \& Chemical Engineering, 32(4), 841-856.

12) Peng, Z., Doroodchi, E. and Evans, G. (2010), DEM simulation of aggregation of suspended nanoparticles, Powder Technology, 204(1), 91-102.

13) Agbangla, G. C., Climent, E. and Bacchin, P. (2014), Numerical investigation of channel blockage by flowing microparticles, Computers \& Fluids, 94, 69-83.

14) Succi, S., d'Humières, D., Qian, Y. H. and Orszag, S. A. (1993), On the small-scale dynamical behavior of lattice BGK and lattice Boltzmann schemes, Journal of scientific computing, 8(3), 219-230.

15) Bhatnagar, P. L., Gross, E. P. and Krook, M. (1954), A model for collision processes in gases. I. Small amplitude processes in charged and neutral one-component systems, Physical review, 94(3), 511.

16) Zhou, J. G. (2009), A lattice Boltzmann method for solute transport, International journal for numerical methods in fluids, 61(8), 848-863.
17) Sullivan, S. P., Sani, F. M., Johns, M. L. and Gladden, L. F. (2005), Simulation of packed bed reactors using lattice Boltzmann methods, Chemical Engineering Science, 60(12), 3405-3418.

18) Kang, Q., Zhang, D., Lichtner, P. C. and Tsimpanogiannis, I. N. (2004), Lattice Boltzmann model for crystal growth from supersaturated solution, Geophysical Research Letters, 31(21).

19) Lu, G., DePaolo, D. J., Kang, Q. and Zhang, D. (2009), Lattice Boltzmann simulation of snow crystal growth in clouds., Journal of Geophysical Research: Atmospheres (1984-2012), 114(D7).

20) Filippova, O. and Hänel, D. (1997), Lattice-Boltzmann simulation of gas-particle flow in filters, Computers \& Fluids, 26(7), 697-712.

21) Yamamoto, K., Takada, N. and Misawa, M. (2005), Combustion simulation with Lattice Boltzmann method in a three-dimensional porous structure, Proceedings of the Combustion Institute, 30(1), 1509-1515 